\title{
EXPLICIT UPPER BOUNDS FOR THE SOLUTIONS OF SOME DIOPHANTINE EQUATIONS
}

\author{
K. GYÖRY
}

\section{Introduction}

Let $L$ be an algebraic number field of degree $l \geqq 1$ with a ring of integers $Z_{L}$. Let $F(\mathbf{x})=F\left(x_{1}, \ldots, x_{m}\right) \in \boldsymbol{Z}_{L}\left[x_{1}, \ldots, x_{m}\right]$ be a decomposable form of degree $n \geqq 3$ in $m \geqq 2$ variables. We may suppose without loss of generality that the coefficient of $x_{1}^{n}$ is not zero (see e.g. [2] and [11]). Suppose that in the factorization

$$
F(\mathbf{x})=a_{0} L_{1}(\mathbf{x}) \ldots L_{n}(\mathbf{x}), \quad 0 \neq a_{0} \in Z_{L},
$$

of $F$ the system $\mathscr{L}$ of the linear forms $L_{j}(\mathbf{x})=x_{1}+\alpha_{2 j} x_{2}+\ldots+\alpha_{m j} x_{m}, j=1, \ldots, n$, is connected (i.e. for any distinct $i, j$ with $1 \leqq i, j \leqq n$ there is a sequence $L_{i}=$ $L_{i_{1}}, \ldots, L_{i_{v}}=L_{j}$ in $\mathscr{L}$ such that for each $u$ with $1 \leqq u \leqq v-1 L_{i_{u}}, L_{i_{u+1}}$ have a linear combination with non-zero algebraic coefficients which belongs to $\mathscr{L}$; cf. [13] or [11]) and that there is no $0 \neq \mathbf{x} \in L^{m}$ for which $L_{j}(\mathbf{x})=0, j=1, \ldots, n^{1)}$. Let $\beta, \pi_{1}, \ldots, \pi_{s}$ be fixed non-zero algebraic integers in $L$ and let $d$ be a positive integer. Assume that $\pi_{1}, \cdots, \pi_{s}$ are not units. In [11] we obtained as a consequence of our main result that the diophantine equation

$$
F(\mathbf{x})=\beta \pi_{1}^{z_{1}} \ldots \pi_{s}^{z_{s}}
$$

has only finitely many solutions in $\mathbf{x} \in \boldsymbol{Z}_{L}^{m}, z_{1}, \ldots, z_{s} \in \boldsymbol{Z}$ with $N_{L / Q}\left(\left(x_{1}, \ldots, x_{m}\right)\right) \leqq d$ and $z_{1}, \ldots, z_{s} \geqq 0$ and that all these solutions can be effectively determined.

In the proof of our theorem in [11] there occurred various constants which were said to be effectively computable, but which in fact were not explicitly calculated. The purpose of the present paper is to derive appropriate values for these constants, and thereby to obtain an explicit upper bound for the sizes of the solutions of (1). Our main result generalizes a recent theorem of Kotov and Sprindžuk [17] concerning the Thue-Mahler equation. Further, it generalizes some results of Győry [6], [7], Győry and Papp [13], [14], [15] and Trelina [25] concerning norm form, discriminant form and index form equations, respectively.

1) If in particular $m=2$, then every binary form $F \in Z_{L}[x, y]$ which has at least three pairwise nonproportional linear forms in its factorization satisfies these conditions. 


\section{Results}

Let $L, F(\mathbf{x})=F\left(x_{1}, \ldots, x_{m}\right), \beta, \pi_{1}, \ldots, \pi_{s}$ and $d$ be defined as above. Denote by $G$ the splitting field of $F(\mathbf{x})$ over $L$ and let $[G: Q]=g,[G: L]=f$. Let $R_{G}$ and $h_{G}$ (resp. $R_{L}$ and $h_{L}$ ) be the regulator and the class number of $G$ (resp. of $L$ ). Let $r$ denote the number of fundamental units in $G$. Put $\left|N_{L / Q}(\beta)\right| \leqq b$ and ${ }^{2)} \mid \overline{a_{0} \alpha_{i j} \mid} \leqq A$ (with $\alpha_{1 j}=1$ for $j=1, \ldots, n$ ). Suppose, for convenience, that in (1) $\left(\pi_{i}\right)=\mathfrak{p}_{i}^{h_{L}}$, $\mathfrak{p}_{1}, \ldots, \mathfrak{p}_{s}$ being distinct prime ideals in $L$ with norms $N\left(\mathfrak{p}_{i}\right)=p_{i}^{f_{i}}, i=1, \ldots, s$. Here $p_{1}, \ldots, p_{s}$ denote rational primes not exceeding $P$. With the above notations we have the following

Theorem 1. Let $L, F\left(x_{1}, \ldots, x_{m}\right), \beta, \pi_{1}, \ldots, \pi_{s}$ and $d$ be as above. Then for every solution of the equation (1) in $x_{1}, \ldots, x_{m} \in \boldsymbol{Z}_{L}, z_{1}, \ldots, z_{s} \in \boldsymbol{Z}$ with $N_{L / Q}\left(\left(x_{1}, \ldots, x_{m}\right)\right) \leqq d$, $z_{1}, \ldots, z_{s} \geqq 0$ there exists a unit $\varepsilon$ in $L$ such that

where

$$
\max \left\{\overline{\left|\varepsilon x_{1}\right|}, \ldots, \overline{\varepsilon x_{m} \mid},\left(p_{1}^{f_{1} z_{1}} \ldots p_{s}^{f_{s} z_{s}}\right)^{h_{L} / l n}\right\} \leqq d^{1 / l} T,
$$

$$
\begin{gathered}
T=\exp \left\{n^{2} C h_{L} R_{L}^{*} P^{g}(\log P)^{5} R_{G} \log ^{3}\left(R_{G}^{*} h_{G}\right)\left(R_{G}+h_{G} \log P\right)^{s f+2} \cdot\right. \\
\left.\cdot\left(R_{G}+s h_{G} \log P+n \log A+\log b\right)\right\} \\
C=(25(\boldsymbol{r}+s f+3) g)^{22 r+13 s f+2 s r f+42}, \quad R_{G}^{*}=\max \left(R_{G}, e\right) \quad \text { and } \quad R_{L}^{*}=\max \left(R_{L}, e\right) .
\end{gathered}
$$

Suppose $\max _{1 \leqq i \leqq s}\left|\overline{\pi_{i}}\right| \leqq \mathscr{P}(\geqq e)$. From Theorem 1 we can easily deduce the following theorem.

Theorem 2. Let $L, F\left(x_{1}, \ldots, x_{m}\right), \beta, \pi_{1}, \ldots, \pi_{s}$ and $d$ be defined as above. Then all solutions of the equation (1) in $x_{1}, \ldots, x_{m} \in \boldsymbol{Z}_{L}, z_{1}, \ldots, z_{s} \in \boldsymbol{Z}$ with $N\left(\left(x_{1}, \ldots, x_{m}\right)\right) \leqq d$, $z_{1}, \ldots, z_{s} \geqq 0$ satisfy

$$
\begin{gathered}
\left.\max \left\{\overline{\left|x_{1}\right|}, \ldots, \overline{\left|x_{m}\right|}\right\} \leqq\left.\overline{\mid \beta}\right|^{1 / n}\left(d^{1 / l} T\right)^{l\left(\frac{s}{h_{L}} \log \mathscr{P}+1\right.}\right), \\
p_{1}^{f_{1} z_{1}} \ldots p_{s}^{f_{s} z_{s}} \leqq\left(d^{1 / l} T\right)^{l n / h_{L} .}
\end{gathered}
$$

Theorem 2 can be regarded as a p-adic analogue of our Theorem 1 in [13].

Our theorems have several consequences. In this paper we restrict ourselves to some applications concerning diophantine equations. Further applications will be given in a separate paper.

We shall state the consequences of Theorem 2 only, but from Theorem 1 one can deduce similar corollaries.

Let $L, \beta, \pi_{1}, \ldots, \pi_{s}$ and $d$ be as above and let $f(x, y) \in \boldsymbol{Z}_{L}[x, y]$ be a binary form of degree $n \geqq 3$. There is an extensive literature on the Thue-Mahler equation

$$
f(x, y)=\beta \pi_{1}^{z_{1}} \ldots \pi_{s}^{z_{s}}
$$

\footnotetext{
2) $\overline{|\gamma|}$ denotes the maximum absolute value of the conjugates of an algebraic number $\gamma$.
} 
and its applications; we refer the reader to the papers of Coates [3], [4], [5], Sprindžuk [22], [23], [24], Kotov [16] and Kotov and Sprindžuk [17] and thence to the literature mentioned there. The best known upper bounds for the solutions of (4) are due to Kotov and Sprindžuk [17]. Their bounds are especially good in terms of $s$. However, in [17] it is assumed that $n \geqq 5$ and the constants corresponding to our constant $C$ are not explicitly calculated in terms of $l$ and $n$.

Corollary 1. Let $f(x, y) \in \boldsymbol{Z}_{L}[x, y]$ be a binary form of degree $n \geqq 3$ with splitting field $G$ over $L$. Suppose that $f(1,0) \neq 0$ and that $f(x, 1)$ has at least three distinct zeros. Then all solutions of the equation (4) in $x, y \in \boldsymbol{Z}_{L}, z_{1}, \ldots, z_{s} \in \boldsymbol{Z}$ with $N((x, y)) \leqq d, z_{1}, \ldots, z_{s} \geqq 0$ satisfy

$$
\max \{\overline{|x|}, \overline{|y|}\} \leqq \overline{\mid \beta}^{1 / n}\left(d^{1 / l} T^{*}\right)^{l\left(\frac{s}{h_{L}} \log \mathscr{P}+1\right)},
$$

where ${ }^{3)}$

$$
p_{1}^{f_{1} z_{1}} \ldots p_{s}^{f_{s} z_{s}} \leqq\left(d^{1 / l} T^{*}\right)^{\ln / h_{L}},
$$

$$
\begin{gathered}
T^{*}=\exp \left\{n^{2} C h_{L} R_{L}^{*} P^{g}(\log P)^{5} R_{G} \log ^{3}\left(R_{G}^{*} h_{G}\right) \cdot\right. \\
\left.\cdot\left(R_{G}+h_{G} \log P\right)^{s f+2}\left(R_{G}+s h_{G} \log P+n \log (2 \overline{|f|})+\log b\right)\right\}
\end{gathered}
$$

with the $C$ defined above.

In terms of $R_{G}, h_{G}$ and $P$ our upper bound in (5) is better than that of Kotov and Sprindžuk [17].

Let $K$ be an extension of degree $n \geqq 3$ of $L$ and let $G$ be the smallest normal extension of $L$ containing $K$. Let $a_{0} N_{K / L}\left(x_{1}+\alpha_{2} x_{2}+\ldots+\alpha_{m} x_{m}\right) \in Z_{L}\left[x_{1}, \ldots, x_{m}\right]$ be a norm form in $m \geqq 2$ variables such that $K=L\left(\alpha_{2}, \cdots, \alpha_{m}\right),\left[L\left(\alpha_{i}\right): L\right]=n_{i} \geqq 3$, $i=2, \ldots, m$, and $n_{2} \ldots n_{m}=n$. Consider the norm form equation

$$
a_{0} N_{K / L}\left(x_{1}+\alpha_{2} x_{2}+\ldots+\alpha_{m} x_{m}\right)=\beta \pi_{1}^{z_{1}} \ldots \pi_{s}^{z_{s}}
$$

with the $\beta, \pi_{1}, \ldots, \pi_{s}$ introduced above. Put $\max \left(\overline{\left|a_{0}\right|}, \overline{\left|a_{0} \alpha_{2}\right|}, \ldots, \overline{a_{0} \alpha_{m}}\right) \leqq A$. When $s=0$, that is, when no $\pi_{1}, \ldots, \pi_{s}$ are specified, Papp and I [13], [15] obtained explicit bounds for the sizes of the solutions of (6). Those bounds depend on $A, \beta$ and certain parameters of $L$ and $K$. As a consequence of Theorem 2 we obtain the following $\mathfrak{p}$-adic generalization of these results.

Corollary 2. Under the above assumptions all solutions of the equation (6) in $x_{1}, \ldots, x_{m} \in \boldsymbol{Z}_{L}, z_{1}, \ldots, z_{s} \in \boldsymbol{Z}$ with $N\left(\left(x_{1}, \ldots, x_{m}\right)\right) \leqq d, z_{1}, \ldots, z_{s} \geqq 0$ satisfy (3).

Our bound established here depends on $G$ instead of $K$. In the case $L=Q$ our Corollary 2 makes effective, for a wide class of norm forms, a recent theorem of Schlickewei [21] on norm form equations.

${ }^{3)}$ As usual, $\overline{|f|}$ denotes the maximum absolute value of the conjugates of the coefficients of the polynomial $f$. 
Let $L, K, G, \beta, \pi_{1}, \ldots, \pi_{s}$ be as above. Let $\alpha_{1}, \ldots, \alpha_{m}$ be $m \geqq 2$ algebraic integers in $K$ such that $K=L\left(\alpha_{1}, \ldots, \alpha_{m}\right)$. Suppose that $1, \alpha_{1}, \ldots, \alpha_{m}$ are linearly independent over $L$ and that $\max _{1 \leqq i \leqq m} \overline{\left|\alpha_{i}\right|} \leqq A$. Generalizing my results obtained in the case $L=Q, s=0$ ([6], [7]), in [14] Papp and I established upper bounds for the solutions of the discriminant form equation

$$
\operatorname{Discr}_{K / L}\left(\alpha_{1} x_{1}+\ldots+\alpha_{m} x_{m}\right)=\beta \pi_{1}^{z_{1}} \ldots \pi_{s}^{z_{s}} \text {. }
$$

Corollary 3. Under the above assumptions all solutions of the equation (7) in $x_{1}, \ldots, x_{m} \in \boldsymbol{Z}_{L}, z_{1}, \ldots, z_{s} \in \boldsymbol{Z}$ with $N\left(\left(x_{1}, \ldots, x_{m}\right)\right) \leqq d, z_{1}, \ldots, z_{s} \geqq 0$ satisfy (3) with $n$ replaced by $n^{3}$.

It is difficult to compare the estimates of our Corollaries 3 and 4 with those obtained in [14] because the bounds derived in [14] depend on certain parameters of $L$ and $K$, but not on $G$. Further, in [14] the constants corresponding the $C$ defined above are not explicitly given ${ }^{4)}$ in terms of $l$ and $n$. Corollaries 3 and 4 give better bounds in $b$ than those obtained in [14]. Since in [14] we derived our estimates in a more direct way (from my results [8], [9] concerning algebraic integers of given discriminant), the estimates of [14] are much better in terms of $A$.

Let again $L, K, G, \beta, \pi_{1}, \ldots, \pi_{s}$ be defined as above. Consider an order $\mathcal{O}$ of the field extension $K / L$ (i.e. a subring of $\boldsymbol{Z}_{K}$ containing $\boldsymbol{Z}_{L}$ that has the full dimension $n$ as a $\boldsymbol{Z}_{L}$-module) and suppose that $\mathcal{O}$ has a relative integral basis of the form $1, \alpha_{1}, \ldots, \alpha_{n-1}$ over $L$. (Such an integral basis exists for a number of orders of $K / L$; see e.g. [1], [18] and [14]). It is easy to see (cf. [14]) that

$\operatorname{Discr}_{K / L}\left(\alpha_{1} x_{1}+\ldots+\alpha_{n-1} x_{n-1}\right)=\left[\operatorname{Ind}_{K / L}\left(\alpha_{1} x_{1}+\ldots+\alpha_{n-1} x_{n-1}\right)\right]^{2} D_{K / L}\left(1, \alpha_{1}, \ldots, \alpha_{n-1}\right)$,

where the decomposable form $\operatorname{Ind}_{K / L}\left(\alpha_{1} x_{1}+\ldots+\alpha_{n-1} x_{n-1}\right) \in Z_{L}\left[x_{1}, \ldots, x_{n-1}\right]$ is called the index form of the basis $1, \alpha_{1}, \ldots, \alpha_{n-1}$ of $\mathcal{O}$ over $L$. Denote by $D_{K / L}(\mathcal{O})$ the principal ideal generated by $D_{K / L}\left(1, \alpha_{1}, \ldots, \alpha_{n-1}\right)$ and suppose $\max _{1 \leqq i \leqq n-1}\left|\alpha_{i}\right| \leqq$ $\leqq A$.

Consider the index form equation

$$
\operatorname{Ind}_{K / L}\left(\alpha_{1} x_{1}+\ldots+\alpha_{n-1} x_{n-1}\right)=\beta \pi_{1}^{z_{1}} \ldots \pi_{s}^{z_{s}} .
$$

In the case $L=Q, s=0$ the author [6], [7] obtained an effectively computable bound for the solutions $x_{1}, \ldots, x_{n-1}$ of (8). Later Trelina [25] generalized this result of the author to arbitrary $s \geqq 1$. Independently of Trelina, Papp and I [14] established an upper bound for the solutions $x_{1}, \ldots, x_{m} \in Z_{L}, z_{1}, \ldots, z_{s} \geqq 0$ in the general case when $s \geqq 1$ and $L$ is an arbitrary but fixed number field. In [25] and [14] the constants corresponding to our constant $C$ are effectively computable but are not explicitly calculated in terms of $l$ and $n$.

\footnotetext{
4) In the special case $s=0$ our constants in [14] and [13] are explicitly computed.
} 
Corollary 4. All solutions of the equation (8) in $x_{1}, \ldots, x_{n-1} \in Z_{L}, z_{1}, \ldots, z_{s} \in \boldsymbol{Z}$ with $N\left(\left(x_{1}, \ldots, x_{n-1}\right)\right) \leqq d, z_{1}, \ldots, z_{s} \geqq 0$ satisfy

where

$$
\begin{gathered}
\max \left\{\overline{\left|x_{1}\right|}, \ldots, \overline{\left|x_{n-1}\right|}\right\} \leqq \overline{\left|D_{K / L}\left(1, \alpha_{1}, \ldots, \alpha_{n-1}\right) \beta^{2}\right|^{1 / n}\left(d^{1 / l} T^{* *}\right)} l\left(\frac{s}{h_{L}} \log \mathscr{P}+1\right), \\
p_{1}^{f_{1} z_{1}} \ldots p_{s}^{f_{s} z_{s}} \leqq\left(d^{1 / l} T^{* *}\right)^{\ln / h_{L}},
\end{gathered}
$$

$$
\begin{gathered}
T^{* *}=\exp \left\{n^{6} C h_{L} R_{L}^{*} P^{g}(\log P)^{5} R_{G} \log ^{3}\left(R_{G}^{*} h_{G}\right) \cdot\right. \\
\cdot\left(R_{G}+h_{G} \log P\right)^{s f+2}\left(R_{G}+s h_{G} \log P+n^{3} \log A+\log \left(b^{2} N\left(D_{K / L}(\mathcal{O})\right)\right)\right\}
\end{gathered}
$$

with the $C$ defined above.

In the special case $L=Q$ (9) is better in terms of $b, P$ and $s$ than the bound obtained by Trelina [25]. On the other hand, her bound is better in $A$ than (9) (because she followed a similar argument to that applied earlier in [6]; see also [14]). In [25] the bound depends on the maximal prime factor and the number of distinct prime factors of $D_{K / Q}(\mathcal{O})$, but not on $D_{K / Q}(\mathcal{O})$. We could easily get a similar result for the equation (8) by taking the prime ideal factorization of $D_{K / L}\left(1, \alpha_{1}, \ldots, \alpha_{n-1}\right)$ in (26) and applying our Corollary 3.

It is evident that our Corollaries 3 and 4 have applications to algebraic integers with given discriminant and given index, respectively. However, in case of algebraic numbers the more direct deductions used in [8] and [9] yield better estimates.

\section{Proofs}

To prove our theorems we need some lemmas. We keep the notations of Section 2. We suppose that there are $r_{1}$ real and $2 r_{2}$ complex conjugate fields to $G$ and that they are chosen in the usual manner: if $\theta$ is in $G$ then $\theta^{(i)}$ is real for $1 \leqq i \leqq r_{1}$ and $\theta^{\left(i+r_{2}\right)}=\overline{\theta^{(i)}}$ for $r_{1}+1 \leqq i \leqq r_{1}+r_{2}$.

Lemma 1. Let $\alpha$ be a non-zero element in $G$ with $\left|N_{G / Q}(\alpha)\right|=M$ and let $v$ be a positive integer. There exists a unit $\varepsilon$ in $G$ such that

$$
|\log | M^{-1 / g}\left(\alpha \varepsilon^{v}\right)^{(i)}|| \leqq \frac{c_{1}^{*} r v}{2} R_{G}, \quad i=1, \ldots, g,
$$

where $c_{1}^{*}=\left(6 \mathrm{rg}^{2} / \log g\right)^{r}$ or $c_{1}^{*}=1$ according as $r \geqq 1$ or $r=0$.

Proof. This is Lemma 3 in [10].

Let $\mathfrak{P}_{1}, \ldots, \mathfrak{P}_{t}$ be distinct prime ideals in $G$ lying above rational primes not exceeding $P$, and let $\mu_{1}, \ldots, \mu_{t}$ be algebraic integers in $G$ such that $\left(\mu_{i}\right)=\mathfrak{P}_{i}^{h_{G}}$, $i=1, \ldots, t$. Let $\beta_{j}=\varkappa_{j} \mu_{1}^{u_{1 j}} \ldots \mu_{t}^{u_{t j}}$, where $0 \neq \varkappa_{j} \in \boldsymbol{Z}_{G}$ with $\left|N_{G / Q}\left(\varkappa_{j}\right)\right| \leqq N$, and let $u_{1 j}, \ldots, u_{t j}$ be non-negative rational integers, $j=1,2,3$. Suppose $\lambda_{1}, \lambda_{2}, \lambda_{3}$ are non-zero algebraic integers in $G$ satisfying $\max _{j} \overline{\left|\lambda_{j}\right|} \leqq H$. 
Lemma 2. If

$$
\lambda_{1} \beta_{1}+\lambda_{2} \beta_{2}+\lambda_{3} \beta_{3}=0
$$

then

$$
\beta_{j}=\sigma \delta_{j},
$$

where $\sigma=\eta \mu_{1}^{a_{1}} \ldots \mu_{t}^{a_{t}}$ with some unit $\eta \in G$ and non-negative rational integers $a_{1}, \ldots, a_{t}$ and $\delta_{j} \in \boldsymbol{Z}_{G}$ such that

$$
\begin{gathered}
\max _{1 \leqq j \leqq 3} \overline{\left|\delta_{j}\right|} \leqq \exp \left\{c_{2}^{*} P^{g}(\log P)^{3} R_{G} \log ^{3}\left(R_{G}^{*} h_{G}\right)\left(R_{G}+h_{G} \log P\right)^{t+2} .\right. \\
\cdot\left(R_{G}+t h_{G} \log P+\log (H N)\right\},
\end{gathered}
$$

where $c_{2}^{*}=(25(r+t+3) g)^{20 r+13 t+2 r t+40}$.

Proof. This is a special case of our Lemma 6 in [12]. The proof of this lemma is based on some explicit estimates of van der Poorten [19] and van der Poorten and Loxton [20].

Proof of Theorem 1. We follow the proof of our theorem established in [11]. It will be assumed that the reader is familiar with the contents of [11], and only a minimal amount of the discussion of that paper will be repeated here.

Writing $\alpha_{i j}^{\prime}=a_{0} \alpha_{i j}$, we have $\alpha_{i j}^{\prime} \in \boldsymbol{Z}_{G}$ for each $i$ and $j$. We shall prove our theorem for the equation

$$
f(\mathbf{x})=a_{0}^{n-1} F(\mathbf{x})=\prod_{j=1}^{n} L_{j}^{\prime}(\mathbf{x})=a_{0}^{n-1} \beta \pi_{1}^{z_{1}} \ldots \pi_{s}^{z_{s}},
$$

where $L_{j}^{\prime}(\mathbf{x})=\alpha_{1 j}^{\prime} x_{1}+\ldots+\alpha_{m j}^{\prime} x_{m}$.

Let $x_{1}, \ldots, x_{m}, z_{1}, \ldots, z_{s}$ be any solution of (14) with $x_{1}, \ldots, x_{m} \in \boldsymbol{Z}_{L}$, $N\left(\left(x_{1}, \ldots, x_{m}\right)\right) \leqq d$ and $z_{1}, \ldots, z_{s} \geqq 0$. Put

$$
\beta_{j}=\alpha_{1 j}^{\prime} x_{1}+\ldots+\alpha_{m j}^{\prime} x_{m}, \quad j=1, \ldots, n .
$$

Let $\mathfrak{P}_{1}, \ldots, \mathfrak{P}_{t}$ be all distinct prime ideals in $G$ lying above $\mathfrak{p}_{1}, \ldots, \mathfrak{p}_{s}$. From (14) we get

$$
\left(\beta_{j}\right)=\mathfrak{A}_{j} \mathfrak{P}_{1}^{U_{1 j}} \ldots \mathfrak{P}_{t}^{U_{t j}}, \quad j=1, \ldots, n,
$$

where the $\mathfrak{A}_{j}$ are integral ideals in $G$ such that $\mathfrak{U}_{1} \ldots \mathfrak{A}_{n} \mid\left(a_{0}^{n-1} \beta\right)$ and the $U_{k j}$ are non-negative rational integers. The definitions and notations given in [11] remain unchanged, except that we now have $\left(\chi_{j}\right)=\mathfrak{A}_{j} \mathfrak{P}_{1}^{r_{1 j}} \ldots \mathfrak{P}_{t}^{r_{t j}}$,

and, by Lemma 1,

$$
\left|N_{G / Q}\left(\chi_{j}\right)\right| \leqq A^{(n-1) g} b^{f} p^{t g h_{G}}
$$

$$
\overline{\left|\mu_{k}\right|} \leqq c_{3}^{*} P_{G}^{h_{G}}, \quad \overline{\left|\chi_{j}\right|} \leqq c_{3}^{*} A^{n-1} b^{f / g} P^{t h_{G}}
$$

for $k=1, \ldots, t$ and $j=1, \ldots, n$, where $c_{3}^{*}=\exp \left\{\left(c_{1}^{*} r / 2\right) R_{G}\right\}$. In [11] we may choose $c_{9}=2 A^{2}$ and we may apply Lemma 2 to (17) of [11]. Then we get

$$
\beta_{q}=\sigma \delta_{q}, \quad \sigma=\varepsilon_{3} \mu_{1}^{a_{1}} \ldots \mu_{t}^{a_{t}},
$$


where $\varepsilon_{3}$ is a unit in $G, a_{1}, \ldots, a_{t} \in \boldsymbol{Z}$ with $a_{k} \geqq 0$ and $\delta_{q} \in \boldsymbol{Z}_{G}$ with

$$
\begin{aligned}
\overline{\left|\delta_{q}\right|} \leqq \exp \left\{2 g c_{2}^{*} P^{g}(\log P)^{3} R_{G} \log ^{3}\left(R_{G}^{*} h_{G}\right)\left(R_{G}+h_{G} \log P\right)^{t+2} .\right. \\
\left.\cdot\left(R_{G}+t h_{G} \log P+n \log A+\log b\right)\right\}=T_{1}, \quad q=1,2,3,
\end{aligned}
$$

in place of (27) of [11].

Continuing the argument of [11], a suitable choice for $c_{36}$ is $n g / h_{G} \log 2$. Further, in view of the above estimates (18), (20) and of (32) of [11] we obtain (34) of [11], that is,

$$
\overline{\left|\tau_{j}\right|} \leqq I_{k=1}^{t} \overline{\mid \mu_{k}} b_{k}^{*} \mid \overline{\varphi_{j} / \psi_{j} \mid} \leqq \exp \left\{c_{37} s \log P \log T_{1}\right\}=T_{2}
$$

with $c_{37}=c_{1}^{*} r n f g R_{G}$.

In place of (35) of [11] we get now from (14)

$$
\left(a_{0}^{n-1} \beta\right) \mathfrak{p}_{1}^{v_{1}} \ldots \mathfrak{p}_{s}^{v_{s}}=\left(\beta_{1} \ldots \beta_{n}\right)=\left(\left(\vartheta \mu_{1}^{d_{1}} \ldots \mu_{t}^{d_{t}}\right)^{n} \tau_{1} \ldots \tau_{n}\right),
$$

where $v_{k}=z_{k} h_{L}$. Formula (21) implies

$$
\operatorname{ord}_{\mathfrak{P}}\left(\prod_{j=1}^{n} \tau_{j}\right) \leqq n g \log T_{2}
$$

for each prime ideal of $G$ lying above $\mathfrak{p}_{k}$. Similarly,

$$
\operatorname{ord}_{\mathfrak{P}}\left(a_{0}^{n-1} \beta\right) \leqq(n-1) g \log A+f \log b .
$$

The argument of [11] applies if we replace in (36) and (37) of [11] $\min \left(v_{k} e_{k}-\operatorname{ord}_{\mathfrak{p}}\left(\prod_{j=1}^{n} \tau_{j}\right), v_{k} e_{k}\right)$ by

$$
\min \left(v_{k} e_{k}+\operatorname{ord}_{\mathfrak{p}}\left(a_{0}^{n-1} \mathfrak{P}\right)-\operatorname{ord}_{\mathfrak{P}}\left(I_{j=1}^{n} \tau_{j}\right), v_{k} e_{k}\right) .
$$

Then we have (38) and (39) of [11] with $c_{39}=h_{L} n g$ and $c_{40}=2 c_{39}$. Let now $p_{1}^{h_{L} y_{1}} \ldots$ $\mathfrak{p}_{s}^{h_{L} y_{s}}=\left(\pi_{1}^{y_{1}} \ldots \pi_{s}^{y_{s}}\right)=(\varkappa)$, where $x \in \boldsymbol{Z}_{L}$, and choose $\xi$ as in [11]. Then (39) of [11] implies that a suitable value for $c_{41}$ is $c_{40} f g=2 h_{L} f g^{2} n$.

Lemma 1 together with (34) and (41) of [11] imply that an appropriate choice for $c_{42}$ is $2 n c_{41} R_{L}^{*} / g$, and it follows immediately that suitable values for $c_{43}$ and $c_{44}$ occurring in (43) and (45) of [11] are given by (3/2) $c_{42}$ and $2 c_{42}$, respectively.

To estimate $\overline{|v|}$ and $\overline{\left|v_{i}\right|}$ we can use Hadamard's inequality. Since $m \leqq n f$, it is easily seen that appropriate choices for $c_{45}$ and $c_{46}$ are $(n f)^{n f / 2} A^{n f}$ and $(n f)^{n f / 2} A^{n f-1}$, respectively. Consequently, $c_{47}$ can be taken as $\left(c_{45}\right)^{g / f} d=(n f)^{n g / 2} A^{n g} d$. In view of Lemma $1 c_{48}$ can obviously be taken as

$$
\left(c_{47}\right)^{1 / l} \exp \left\{\left(c_{1}^{*} r / 2\right) R_{L}\right\}=(n f)^{n g / 2 l} A^{n g / l} d^{1 / l} \exp \left\{\left(c_{1}^{*} r / 2\right) R_{L}\right\} .
$$

Finally, in (52) of [11] a suitable choice for $c_{49}$ is $c_{48} c_{46} c_{45}^{l-1}$, which is less than

$$
(n f)^{2 n g} A^{2 n g} d^{1 / l} \exp \left\{\frac{c_{1}^{*} r}{2} R_{L}\right\} .
$$


So, by (52) of [11] we have

$$
\begin{gathered}
\max _{1 \leqq i \leqq m} \overline{\left|x_{i}^{\prime}\right|}<c_{49} T_{3}=c_{49} \exp \left\{8 n f g h_{L} R_{L}^{*} s \log P \log T_{2}\right\} \\
=c_{49} \exp \left\{8 r c_{1}^{*}(f g n)^{2} h_{L} R_{L}^{*} s^{2}(\log P)^{2} \log T_{1}\right\} \\
=d^{1 / l} \exp \left\{16 r(s f n)^{2} g^{3} c_{1}^{*} c_{2}^{*} h_{L} R_{L}^{*} P^{g}(\log P)^{5} R_{G} \log ^{3}\left(R_{G}^{*} h_{G}\right) \cdot\right. \\
\left.\cdot\left(R_{G}+h_{G} \log P\right)^{s f+2}\left(R_{G}+s h_{G} \log P+n \log A+\log b\right)\right\} .
\end{gathered}
$$

Since $16 r(s f)^{2} g^{3} c_{1}^{*} c_{2}^{*}<(25(r+s f+3) g)^{22 r+13 s f+2 r s f+42}$, (23) provides the desired upper bound for $\max _{1 \leqq i \leqq m} \overline{\left|x_{i}^{\prime}\right|}$.

Finally, it follows from (44), (45) and (50) of [11] that

$$
\begin{gathered}
\left(p_{1}^{f_{1} z_{1}} \ldots p_{s}^{f_{s} z_{s}}\right)^{f h_{L}} \leqq\left|N_{G / Q}\left(a_{0}^{n-1} \beta \pi_{1}^{z_{1}} \ldots \pi_{s}^{z_{s}}\right)\right|=\left|N_{G / Q}\left(\beta_{1} \ldots \beta_{n}\right)\right| \\
=\left|N_{G / Q}(\varkappa)\right|^{n}\left|N_{G / Q}\left(\xi_{1} \ldots \xi_{n}\right)\right| \leqq c_{47}^{n f} T_{3}^{n g},
\end{gathered}
$$

and this completes the proof of Theorem 1.

Proof of Theorem 2. Let $x_{1}, \ldots, x_{m}, z_{1}, \ldots, z_{s}$ be an arbitrary but fixed solution of (1) with the given properties. By Theorem 1 there exists a unit $\varepsilon$ for which (2) holds. Further, from (1) we get

$$
F\left(\varepsilon x_{1}, \ldots, \varepsilon x_{m}\right)=\varepsilon^{n} \beta \pi_{1}^{z_{1}} \ldots \pi_{s}^{z_{s}} .
$$

But it follows from (2) that

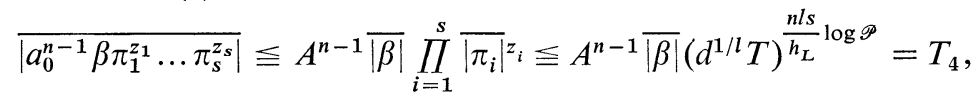

where $T$ denotes the expression occurring in (2). On the other hand, by (14) we have

$$
\overline{\left|a_{0}^{n-1} F\left(\varepsilon x_{1}, \ldots, \varepsilon x_{m}\right)\right|} \leqq\left(m A d^{1 / l} T\right)^{n} \leqq\left(n f A d^{1 / l} T\right)^{n}=T_{5} .
$$

Thus we obtain from (24)

whence

$$
\overline{\left|\varepsilon^{-n}\right|}=\overline{\left|\varepsilon^{-1}\right|^{n}} \leqq T_{4} \cdot T_{5}^{l-1} \leqq \overline{|\beta|}(n f)^{n(l-1)} A^{n l-1}\left(d^{1 / l} T\right)^{\frac{n l s}{h_{L}} \log \mathscr{P}+n(l-1)},
$$

$$
\overline{\left|\varepsilon^{-1}\right|} \leqq \overline{|\beta|^{1 / n}}(n f)^{l-1} A^{l}\left(d^{1 / l} T\right)^{\frac{l s}{h_{L}} \log \mathscr{P}+(l-1)} .
$$

Finally (2) and (25) imply (3).

Proof of Corollary 1. In what follows it will again be supposed that the reader is familiar with the proofs of the corollaries occurring in [11]. By assumption there are at least three pairwise nonproportional linear factors in the factorization

$$
f(x, y)=a_{0}\left(x+\alpha_{1} y\right) \ldots\left(x+\alpha_{n} y\right) .
$$

So, in order to apply our Theorem 2 it suffices to give an upper bound for $\max \left(\overline{\left|a_{0}\right|}, \overline{\left|a_{0} \alpha_{1}\right|}, \ldots, \overline{\left|a_{0} \alpha_{n}\right|}\right)$. But it is known that $\overline{\left|a_{0} \alpha_{i}\right|}<\overline{a_{0} \mid}+\overline{|f|} \leqq 2|\bar{f}|$; hence (5) follows from (3). 
Proof of Corollary 2. The argument of the proof of Corollary 4 of [11] shows that the equation (6) satisfies all the conditions of our Theorem 2. This proves the required assertion.

Proof of Corollary 3. As is known, there exists $\alpha=\alpha_{1}+a_{2} \alpha_{2}+\ldots+a_{m} \alpha_{m}$ with $D_{K / L}(\alpha) \neq 0, a_{i} \in \mathbf{Z}_{L}, \quad \overline{a_{i} \mid} \leqq n^{4}, \quad i=2, \ldots, m$. Let $x_{1}=x_{1}^{\prime}, \quad x_{i}=a_{i} x_{1}^{\prime}+x_{i}^{\prime}, \quad i=2, \ldots, m$. Then (7) gives $\operatorname{Discr}_{K / L}\left(\alpha x_{1}^{\prime}+\alpha_{2} x_{2}^{\prime}+\ldots+\alpha_{m} x_{m}^{\prime}\right)=\beta \pi_{1}^{z_{1}} \ldots \pi_{s}^{z_{s}}$. By applying Theorem 2 to this equation our statement follows (cf. [11]).

Proof of Corollary 4. Every solution of (8) satisfies

$$
\operatorname{Discr}_{K / L}\left(\alpha_{1} x_{1}+\ldots+\alpha_{n-1} x_{n-1}\right)=D_{K / L}\left(1, \alpha_{1}, \ldots, \alpha_{n-1}\right) \beta^{2} \pi_{1}^{2 z_{1}} \ldots \pi_{s}^{2 z_{s}} .
$$

Now Corollary 3 applies and (9) follows immediately.

\section{References}

[1] Berwick, W. E. H.: Integral bases. - Reprinted by Stechert-Hafner Service Agency, New York-London, 1964.

[2] Borevich, Z. I., and I. R. Shafarevich: Number theory. - Academic Press, New York-London, 1967.

[3] CoAtes, J.: An effective $p$-adic analogue of a theorem of Thue. - Acta Arith. 15, 1969, 279305.

[4] CoAtes, J.: An effective $p$-adic analogue of a theorem of Thue II. The greatest prime factor of a binary form. - Acta Arith. 16, 1970, 399-412.

[5] Contes, J.: An effective $p$-adic analogue of a theorem of Thue III. The diophantine equation $y^{2}=x^{3}+k$. - Acta Arith. 16, 1970, 425-435.

[6] GYöRY, K.: Sur les polynômes à coefficients entiers et de discriminant donné, III. - Publ. Math. Debrecen 23, 1976, 141-165.

[7] Győry, K.: Polynomials with given discriminant. - Colloquia Mathematica Societatis János Bolyai 13, Debrecen, 1974, Topics in number theory, edited by P. Turán, North-Holland Publishing Company, Amsterdam-Oxford-New York, 1976, 65-78.

[8] GYŐRY, K.: On polynomials with integer coefficients and given discriminant, IV. - Publ. Math. Debrecen 25, 1978, 155-167.

[9] GYŐRY, K.: On polynomials with integer coefficients and given discriminant, V. p-adic generalizations. - Acta Math. Acad. Sci. Hungar. 32, 1978, 175-190.

[10] GYÖRY, K.: On the solutions of linear diophantine equations in algebraic integers of bounded norm. - Ann. Univ. Sci. Budapest. Eötvös Sect. Math. (to appear).

[11] GYöRY, K.: On the greatest prime factors of decomposable forms at integer points. - Ann. Acad. Sci. Fenn. Ser. A I 4, 1978/1979, 341-355.

[12] GYöRY, K.: On the number of solutions of linear equations in units of an algebraic number field. - Comment. Math. Helv. 54, 1979, 583-600.

[13] GYŐRY, K., and Z. Z. PAPP: Effective estimates for the integer solutions of norm form and discriminant form equations. - Publ. Math. Debrecen 25, 1978, 311-325.

[14] GyörY, K., and Z. Z. PAPP: On discriminant form and index form equations. - Studia Sci. Math. Hungar. (to appear).

[15] GYŐRY, K., and Z. Z. PAPP: Norm form equations and explicit lower bounds for linear forms with algebraic coefficients. - To appear. 
[16] Котоv, S. V.: The Thue-Mahler equation in relative fields. - Collection of articles in memory of Juriŭ Vladimirovič Linnik, Acta Arith. 27, 1975, 293-315 (Russian).

[17] Kotov, S. V., and V. G. SPRINDžUK: The Thue-Mahler equation in relative fields and approximation of algebraic numbers by algebraic numbers. - Izv. Akad. Nauk SSSR Ser. Mat. 41, 1977, 723-751 (Russian).

[18] Narkiewicz, W.: Elementary and analytic theory of algebraic numbers. - PWN-Polish Scientific Publishers, Warszawa, 1974.

[19] VAn der Poorten, A. J.: Linear forms in logarithms in the $p$-adic case. - Transcendence Theory: Advances and Applications, edited by A. Baker and D. W. Masser, Academic Press, London-New York-San Francisco, 1977, 29-57.

[20] van der Poorten, A. J., and J. H. Loxton: Multiplicative relations in number fields. - Bull. Austral. Math. Soc. 16, 1977, 83-98 and 17, 1977, 151-156.

[21] Schlickewer, H. P.: On norm form equations. - J. Number Theory 9, 1977, no. 3, 370380.

[22] SprindžUK, V. G.: A new application of p-adic analysis to representations of numbers by binary forms. - Izv. Akad. Nauk SSSR Ser. Mat. 34, 1970, 1038-1063 (Russian).

[23] SprindžUk, V. G.: The rational approximations to algebraic numbers. - Izv. Akad. Nauk SSSR Ser. Mat. 35, 1971, 991-1007 (Russian).

[24] SPRIndžUk, V. G.: The structure of numbers representable by binary forms. - Dokl. Akad. Nauk BSSR 17, 1973, 685-688, 775 (Russian).

[25] Trelina, L. A.: On the greatest prime factor of an index form. - Dokl. Akad. Nauk BSSR 21, 1977, 975-976 (Russian).

Kossuth Lajos University

Mathematical Institute

H-4010 Debrecen 10

Hungary

Received 26 January 1979 